affected, continued to report subjective feelings of impairment throughout most of the two postoperative days. The group who breathed spontaneously, on the other hand, had subjectively recovered to preoperative levels by the middle of the first day after the operation, but their performance was still impaired during the second day. This divergence between subjective and objective estimates of recovery is important and suggests that patients should be advised not to rely on their own judgment of fitness to drive. It should be pointed out to them that some aspects of mental ability may remain impaired even after they feel that they have made a full recovery.

We thank Trent Regional Health Authority for financial support and the ward staff for their help in this study.

\section{References}

${ }^{1}$ Havard JA. Medical aspects of fitness to drive. London: Medical Commission on Accident Prevention, 1976.

2 Baskett PJF, Vickers MD. General anaesthesia and driving. Lancet $1979 ; \mathrm{i}: 490$.
${ }^{3}$ Kortilla K, Linnoila M, Ertama P, Hakkinen S. Recovery and simulated driving after intravenous anaesthesia with thiopental, methohexital, propanidid or alphadione. Anesthesiology 1975;43:291-8.

' Epstein BS. Recovery from anesthesia. Anesthesiology $1975 ; 43: 285-8$.

${ }^{5}$ Herbert $M$. Assessment of performance in studies of anaesthetic agents. Br F Anaesth 1978;50:33-8.

${ }^{6}$ Wilkinson RT, Houghton D. Portable four-choice reaction time test with magnetic tape memory. Behavior Research Methods and Instrumentation $1975 ; 7: 441$.

${ }^{7}$ Herbert M, Johns MW, Dore C. Factor analysis of analogue scales measuring subjective feelings before and after sleep. Br $\mathcal{F ~ M e d ~ P s y c h o l ~}$ 1976;49:373-9.

${ }^{*}$ Herbert M, Bourke JB, Rose JM. The assessment of preoperative medication by a visual analogue technique. In: Oborne DJ, Gruneberg MM, Eiser JR, eds. Research in psychology and medicine. Vol 1. London: Academic Press, 1979.

${ }^{9}$ Jeffcoate WJ, Herbert $M$, Cullen $M H$, Hastings AG, Walder CP. Prevention of effects of alcohol intoxication by naloxone. Lancet 1979 ;ii 1157-9.

1" Scott A, Whitnam JG, Wilkinson RT, Whitten JEJ. Assessment of postoperative performance decrement using a serial four-choice reaction-time test. Proceedings of the Anaesthetic Research Society. Br J Anaesth 1980;52:629-30.

\title{
Method of sampling chorionic villi in first trimester of pregnancy under guidance of real time ultrasound
}

\author{
R H T WARD, B MODELL, M PETROU, F KARAGÖZL $\bar{U}$, E DOURATSOS
}

\begin{abstract}
Samples of chorionic villi were obtained in the first trimester by aspiration using a cannula passed transcervically under the guidance of real time ultrasound. In initial studies in $\mathbf{4 7}$ anaesthetised patients immediately before therapeutic abortion a method was developed giving a success rate of $89 \%$. In 10 patients successful sampling was performed as an outpatient procedure without anaesthesia. In all, seven diagnostic procedures were undertaken and four of the five unaffected pregnancies continued.

The technique of chorionic villous sampling using real time ultrasound is simple to learn and yields material for biochemical analysis and chromosomal study without the need for tissue culture. The exact obstetric risk, however, remains to be defined.
\end{abstract}

\section{Introduction}

The development of DNA analysis for fetal diagnosis, particularly of the haemoglobinopathies, ${ }^{1}$ provided the impetus to develop a simple and safe method of obtaining chorionic villous material early in pregnancy. In China blind sampling of

\footnotetext{
Department of Obstetrics and Gynaecology, School of Medicine, University College London, London WC1E 6AU

R H T WARD, MA, FRCOG, consultant and senior lecturer

B MODELL, MA, MRCPATH, consultant in perinatal medicine

M PETROU, BSC, research assistant

F KARAGÖZL $\bar{U}$, MD (Istanbul), research fellow

E DOURATSOS, MD (Athens), research fellow

Correspondence to: $\mathrm{Mr} \mathrm{R} \mathrm{H} \mathrm{T}$ Ward.
}

chorionic villi for determining the sex of the fetus reportedly has a low complication rate. ${ }^{2}$ In Russia a method using flexible biopsy forceps under ultrasound guidance has also been devised for fetal diagnosis of genetic sex linked conditions, and all 13 pregnancies that were allowed to continue after the procedure had a normal outcome. ${ }^{3}$

Mid-trimester diagnosis for the haemoglobinopathies by fetal blood sampling at fetoscopy carries an appreciable risk for the fetus $^{4}$ and may result in emotional and physical trauma associated with late therapeutic abortion in the $25 \%$ of cases in which this is indicated. A diagnostic technique for use in the first trimester would have the major advantage that termination of an affected pregnancy, when requested, could be undertaken under general anaesthesia at a safe and acceptable time for the patient.

A gene mapping method used for first trimester fetal diagnosis in three cases at risk for haemoglobinopathies has been described. ${ }^{5}$ In this paper we describe the obstetric technique and outline some of the problems encountered in early chorionic villous sampling.

\section{Patients and methods}

Initially the feasibility of obtaining chorionic villi was tested in anaesthetised patients before termination in the first trimester. In phase 1 (28 patients) either a 16 gauge Medicut cannula or a specially designed Portex cannula with one or more side holes was used, with suction provided by a $2 \mathrm{ml}$ or $5 \mathrm{ml}$ syringe. The siting of the cannula was monitored with linear array real time ultrasound (Toshiba SAL-20A) and samples examined in the laboratory under a dissecting microscope for the presence of villous material. In phase $2(19$ patients) the Portex cannula had an end hole and an aluminium obturator which could be curved appropriately and is visible in the ultrasound scan. A $20 \mathrm{ml}$ syringe provided greater suction. The presence of villous material was confirmed histologically. In phase 3 (14 patients) successful chorionic villous sampling was confirmed in 
the operating theatre using a dissecting microscope and samples sent for DNA extraction to confirm that adequate material was being obtained. In order to obtain short term follow up of the effects of sampling on pregnancy we asked three of the 14 women for permission to take samples in the outpatient department five days before their scheduled operation for termination of pregnancy.

Informed consent was obtained from all the patients and the study was approved by the hospital's ethics committee. The last seven patients in phase 3 requested the procedure for fetal diagnosis and intended that their pregnancies should continue if the fetus proved healthy.

\section{OBSTETRIC TECHNIQUE}

Couples requesting a diagnostic procedure are carefully counselled before we agree to undertake chorionic villous sampling. In this series they were told that our experience of the technique was limited and that it had not been used in pregnancies intended to continue.

At the first visit a detailed ultrasound examination is performed in which viability (as assessed from fetal movements and heart rate) and gestational age (by measurement of crown-rump length) are confirmed and multiple pregnancy excluded. Sampling is carried out between eight and 12 weeks of pregnancy, when the ultrasound examination is repeated and the chorion frondosum located-that is the definitive placenta (fig 1). The chorion frondosum is seen as an area of increased reflectivity (brightness). The patient is placed in the lithotomy position and, after vaginal toilet with aqueous chlorhexidine, bimanual examination performed. The cervix is then visualised with a Sims speculum and the external os cleaned. Usually no tenaculum is required to steady the cervix, although for an acutely anteflexed and anteverted uterus it may be necessary to apply gentle downward traction on the cervix.

The sampling cannula is made of soft polyethylene $1.5 \mathrm{~mm}$ diameter with an overall length of $17 \mathrm{~cm}$ (fig 2). A removable blunt ended aluminium obturator protrudes sufficiently to obliterate the open end. The assembled cannula is bent for satisfactory intrauterine positioning as judged from the site of the chorion frondosum and the distance from the internal os. The "stop" is then positioned to avoid overshooting the site to be sampled.

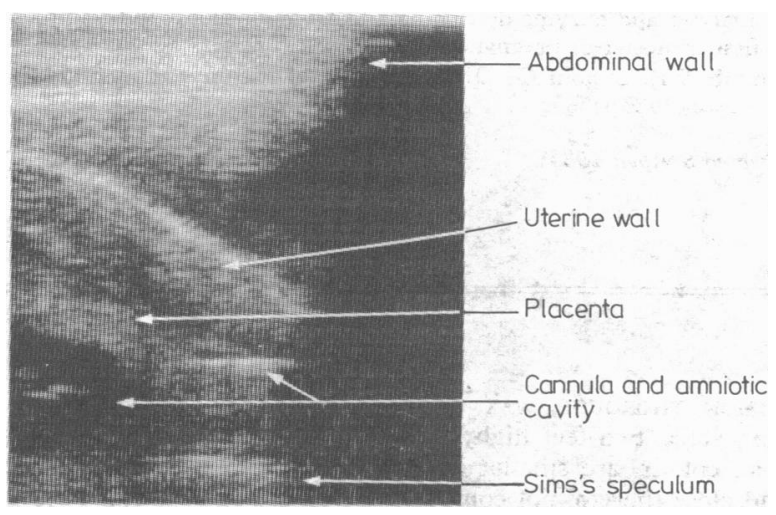

FIG 1-Longitudinal real time scan at 12 weeks of pregnancy showing cannula at sampling site.

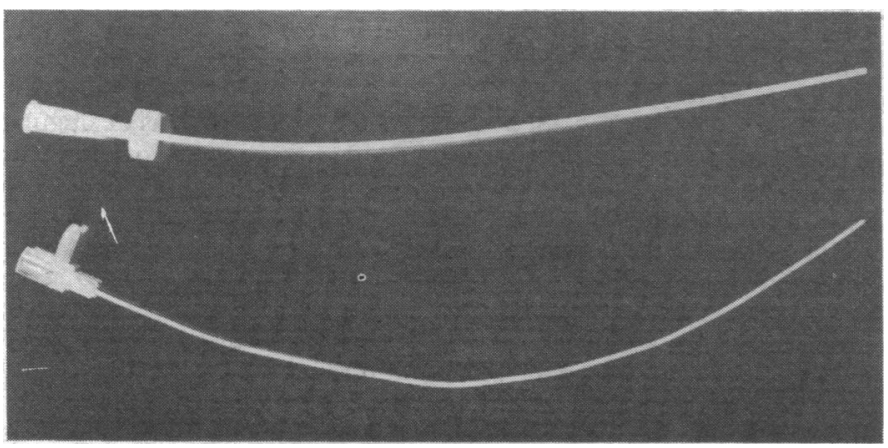

FIG 2-Sampling cannula and obturator.
Usually we find the developing placenta situated on the posterior uterine wall, so that after passing the cannula through the cervical canal to the level of the internal os, with the concavity anteriorly, rotation through 180 degrees permits easy access to the chosen site. The tip is guided to the lowest border of the chorion frondosum. During this final movement the gestational sac is sometimes indented. The cannula is held firmly while the obturator is removed and replaced with a $20 \mathrm{ml}$ syringe. Five to $10 \mathrm{ml}$ of suction is applied, and with a longitudinal scan tissue movement up the cannula may be observed, sometimes leaving a small echo free area at the sampling site. The entire cannula is withdrawn, still with suction applied to minimise the possibility of infection. The outside of the cannula is wiped clean of any cervical mucus or streaks of maternal blood before being flushed into culture medium. The sample is then examined under a low power dissecting microscope in a Petri dish.

With a little practice chorionic villous material is easily recognised. When enough is present the procedure is discontinued, but if none or an inadequate amount has been obtained the procedure is repeated, placing the tip of the cannula in a slightly different site. Usually one to three samples are taken, but the procedure has been repeated up to five times with no obvious untoward effect.

The procedure causes little discomfort and is usually completed within five minutes. No cervical dilatation has been necessary and a uterine sound is not used. The patient is allowed to return home shortly afterwards, and repeat ultrasound examination is arranged within two to four days to reassure both the patient and the obstetrician of continuing fetal viability. In $\mathrm{Rh}$ negative patients $50 \mu \mathrm{g}$ anti-D has been given.

\section{Results}

The table shows the results of sampling in all 66 women between seven and 14 weeks of pregnancy. In phase 1 a chorionic villous sample

Results of sampling chorionic villi in 66 women between seven and 14 weeks of gestation

\begin{tabular}{cccc} 
Phase & $\begin{array}{c}\text { No of patients } \\
\text { (total = 66) }\end{array}$ & $\begin{array}{c}\text { Gestation } \\
\text { (weeks) }\end{array}$ & $\begin{array}{c}\text { Trophoblast obtained } \\
\text { (see text) }\end{array}$ \\
\hline 1 & $26^{*}$ & $7-12$ & $8(31 \%)$ \\
2 & 19 & $7-13$ & $17(89 \%)$ \\
3 & $21 \dagger$ & $7-14$ & $19(90 \%)$ \\
\hline
\end{tabular}

* Two other patients were found to have had missed abortions. †Includes three outpatient procedures before termination of pregnancy and seven diagnostic procedures.

was obtained in only eight of 26 patients. Two other patients were found to have had missed abortions, diagnosed by the absence of a fetus on ultrasound and absence of fetal material on termination of pregnancy. Chorionic villi, however, were readily obtained in both cases. In phases 2 and 3 the success rates were 17 out of 19 cases $(89 \%)$ and 19 out of 21 cases $(90 \%)$ respectively, and the total nucleic acid recovered ranged from 10 to $100 \mu \mathrm{g}$ (mean $50 \mu \mathrm{g}$ ). Details of the fetal diagnoses are given elsewhere. ${ }^{5}$ In the two cases in phase 3 in which samples were not obtained the microscope was not available in the theatre. Of the 10 patients in phase 3 in whom outpatient sampling was performed, one (diagnostic) showed a missed abortion two days later. Severe cervicitis had been present at the time of the procedure at eight weeks. In three patients pregnancy was terminated as planned five days later. In two diagnostic cases the fetus was shown to have homozygous thalassaemia, and three weeks later the pregnancies were terminated by suction under general anaesthesia. In both cases pure fetal blood obtained from the fetal cord after abortion allowed confirmation of the diagnosis by conventional biochemical methods. In the remaining four diagnostic cases the pregnancies continued.

\section{Discussion}

Before agreeing to undertake diagnostic chorionic villous sampling in pregnancies that were intended to continue we had to achieve an acceptable success rate in obtaining a suitable amount of tissue. The initially poor success improved progressively with modifications in the design of the cannula, with 
experience in real time ultrasound, with the introduction of the microscope in the theatre to give immediate feedback, and with the increasing confidence of the obstetrician. Our objective was to develop a simple and inexpensive technique suitable for diagnosing haemoglobinopathies in developing as well as developed countries. Ultrasound remains the single costly but essential item.

In the early work using a Medicut cannula there were three difficulties: inability to negotiate the cervical canal, inaccessibility of the chorion frondosum, and inadvertent rupture of the gestation sac. These problems were solved by lengthening the cannula, including a flexible obturator with a rounded tip that protrudes slightly from the end of the cannula, and fitting a movable "stop."

We deliberately try to sample from the edge of the definitive placenta or chorion frondosum. While it is tempting to sample chorion laeve, which is destined to degenerate, samples are usually small and atrophic changes are often present, so that the material may not be suitable for chromosomal or DNA analysis.

We believe that nine to 10 weeks is the optimum time for sampling. In common with others, ${ }^{6}$ we have the highest success in obtaining an adequate sample at the first attempt between eight and 10 weeks. Nevertheless, organogenesis is only just complete by eight weeks, so that sampling earlier than this may carry a risk of teratogenesis. Sampling after 11 weeks is also suboptimal because at present a diagnosis based on gene mapping takes 10-20 days, and if an abortion is needed after 14 weeks it must be done using a mid-trimester procedure with its inherent disadvantages.

The risks that should be anticipated include accidental abortion, infection, and bleeding. It is difficult to assess the true risk of abortion, since a realistic spontaneous abortion rate between eight and 12 weeks is roughly $10 \%$. Therefore as the method becomes more widely used for diagnosis a collaborative international study will be needed.

Local infection associated with cervicitis may have been the main factor in the one pregnancy that ended in abortion after sampling. Very slight spotting of blood the next day was noted by two of the 10 women whose pregnancies were followed up for some time. As fetomaternal haemorrhage may occur as a result of sampling, we now administer anti-D to all $R h$ negative patients.

Four of the seven women requesting diagnostic procedures had previously undergone mid-trimester fetal blood sampling, and two of these had then had a termination. One of the seven had previously had a first trimester abortion rather than accept mid-trimester diagnosis. Only one was a primigravida. All requested a first trimester procedure, fully understanding the risks. Their response suggests that even if chorionic villous sampling proves to carry significantly greater risks than fetoscopy, many patients would prefer first trimester diagnosis to avoid late abortion of a fetus with whom bonding is already established. We plan to continue our diagnostic series in order to clarify all these issues.

The obstetric method is simple and the material obtained may be used directly without tissue culture for biochemical analysis ${ }^{3}$ and chromosomal studies. ${ }^{7}$ This makes it a highly desirable approach to offering a genetic service in developing countries and confers great cost-benefit as well as humanitarian advantages in advanced communities. Probably the associated DNA technology will be simplified in the near future, thus extending the applicability of the method still further.

The obstetric technique is relatively easy to learn. It requires a team of three members-an obstetrician, an ultrasound operator, and an assistant to identify the chorionic villous material using a microscope. The diagnostic team needs to see the procedure only once and can then practise it at terminations of pregnancy until proficiency is reached. It is easy to learn to recognise the chorionic villous material, but an experienced obstetrician and ultrasound operator are needed, as for amniocentesis. Because of the simplicity of the method there is a risk that it might come into widespread use before the obstetric risk has been properly defined. Hence it is essential for all groups to use it as a diagnostic procedure in collaboration with the centralised international recording and follow up system proposed by the hereditary diseases section of the World Health Organisation.

We are grateful for the enthusiasm of the nursing staff of the operating theatre and wards at the National Temperance Hospital and the gynaecological outpatient clinic and labour ward at University College Hospital, and the advice and encouragement of Professor D V I Fairweather, Professor R Williamson, and Dr Dulcie Coleman. We also thank Dr Coleman for the histological studies of the specimens obtained. We acknowledge the cooperation of Dr W Watson, Portex Ltd, and Dr I Sutherland, National Institute for Medical Research, for their help in developing the cannula. The work is sponsored by the World Health Organisation. BM is supported by a generous donation from Prince Sultan bin Abdul Aziz, of Saudi Arabia; MP is supported by the UK Thalassaemia Society; FK is the recipient of a WHO training fellowship; and ED is the recipient of a NATO fellowship.

\section{References}

${ }^{1}$ Williamson R, Eskdale J, Coleman DV, Niazi N, Loeffler FE, Modell B. Direct gene analysis of chorionic villi: a possible technique for firsttrimester antenatal diagnosis of haemoglobinopathies. Lancet 1981; ii:1125-7.

2 Anshan Department of Obstetrics and Gynecology. Fetal sex prediction by sex chromatin of chorionic villi cells during early pregnancy. Chin Med f 1975;1:117-26.

${ }^{3}$ Kazy Z, Rozovsky IS, Bakharev VA. Chorion biopsy in early pregnancy a method for early prenatal diagnosis for inherited disorders. Prenatal Diagnosis $1982 ; 2: 39-45$.

${ }^{4}$ Ward RHT, Modell B, Fairweather DVI, Shirley IM, Richards BA, Hetherington CP. Obstetric outcome and problems of mid-trimester fetal blood sampling for antenatal diagnosis. $\mathrm{Br} \mathcal{F}$ Obstet Gynaecol $1981 ; 88: 1073-80$.

${ }^{5}$ Old JM, Ward RHT, Karagözlū F, Petrou M, Modell B. First-trimester fetal diagnosis for haemoglobinopathies: three cases. Lancet 1982;ii : 1413-6.

${ }^{6}$ Simoni G, Brambati B, Danesino C, et al. Efficient direct chromosome analyses and enzyme determinations from chorionic villi samples in the first trimester of pregnancy. Hum Genet (in press).

${ }^{7}$ Brambati B, Simoni G. Diagnosis of fetal trisomy 21 in first trimester. Lancet $1983 ; \mathrm{i}: 586$.

(Accepted 6 April 1983)

COMMON THOROUGH WAX OR THOROUGH LEAF sends forth a strait round stalk, two feet high, or better, whose lower leaves being of a bluish colour, are smaller and narrower than those up higher, and stand close thereto, not compassing it; but as they grow higher, they do not encompass the stalks, until it wholly pass through them, branching toward the top into many parts, where the leaves grow smaller again, every one standing singly, and never two at a joint. The flowers are small and yellow, standing in tufts at the heads of the branches, where afterwards grow the seed, being blackish, many thick thrust together. The root is small, long and woody, perishing every year, after seed-time, and rising again plentifully of its own sowing. It is found growing in many corn-fields and pasture grounds in this land. It flowers in July, and the seed is ripe in August.

This is under the influence of Saturn. Thorough-Wax is of singular good use for all sorts of bruises and wounds either inward or outward; and old ulcers and sores likewise, if the decoction of the herb with water and wine be drank, and the place washed therewith, or the juice of the green herb bruised, or boiled, either by itself, or with other herbs, in oil or hog's grease, to be made into an ointment to serve all the year. The decoction of the herb, or powder of the dried herb, taken inwardly, and the same, or the leaves bruised, and applied outwardly, is singularly good for all ruptures and burstings, especially in children before they be too old. Being applied with a little flour and wax to children's navels that stick forth, it helps them. (Nicholas Culpeper (1616-54) The Complete Herbal, 1850.) 